EESTI NSV TEADUSTE AKADEEMIA TOIMETISED, 29. KOIDE FUOSIKA * MATEMAATIKA, 1980, NR. 2

ИЗВЕСТИЯ АКАДЕМИИ НАУК ЭСТОНСКОИ ССР. ТОМ 29 ФИЗИКА * МАТЕМАТИКА. 1980, № 2

\title{
R. LEPP, V. OLMAN
}

\section{AN INEQUALITY FOR INTEGRALS WITH SPHERICALLY SYMMETRIC FUNCTIONS AND ITS APPLICATION TO OPTIMIZATION}

\author{
(Presented by N. Alumäe)
}

1. Introduction. Several authors have studied the variation of the value of an integral over a convex symmetric set, the last being translated in a given direction $\left[{ }^{1-3}\right]$. In the present paper we consider a different problem: the variation of the integral while the integration domain is rotated around some fixed point. More precisely, let $f(x)$ be a non-negative Lebesgue integrable function, $x \in R^{n}$, and $M-$ a Borel set in $R^{n}$. The question is how the value

$$
R(a, A)=\int_{A} f(x) d x
$$

depends on an $n$-dimensional orthogonal matrix $A$ and a vector $a \in R^{n}$.

In the next section we treat the two-dimensional case; the results are generalized in Section 3 for the $n$-dimensional case and applied in Section 4 to a quadratic stochastic programming problem.

2. Two-dimension a 1 c a s e. Let $M \subset R^{2}$ be a symmetric set relative to both axes $O X$ and $O Y$, i. e. if $(x, y) \in M$, then $\left(x^{\prime}, y^{\prime}\right) \in M$ for each $\left|x^{\prime}\right|=|x|$ and $\left|y^{\prime}\right|=|y|$. Let us introduce polar coordinates $(\varrho, \alpha): x=$ $=\mathrm{Q} \cos \alpha$ and $y=\mathrm{Q} \sin \alpha$. Suppose that in these coordinates the set $M$ can be described in the following way:

$$
M=\{(\varrho, \alpha): \varrho \leqslant \varphi(\alpha), \quad \varrho \geqslant 0, \quad 0<\alpha<2 \pi\} .
$$

By the symmetry of $M$ we get $\varphi(\alpha)=\varphi(-\alpha)$ and $\varphi(\alpha+\pi)=\varphi(\alpha)$, i. e. $\varphi(\alpha)$ is an even periodic function with period of $\pi$.

Definition 1. A symmetric set $M \subset R^{2}$ is called strongly prolate in $O X$-direction if the function $\varphi(\alpha)$ in (1) is non-increasing on $(0, \pi / 2)$. Strong prolation in L-direction is defined in the same sense with regard to orthogonal rotation of axes so that $L$ coincides with $O X$.

Example. Ellipse is strongly prolate along its long axis.

Theorem 1. Let $g(t)$ be a non-negative, convex and differentiable function on $(0, \infty)$ and $M$ be a set strongly prolate in OX-direction. Then for every $r>0$ the function

$$
H(\theta)=\int_{M+a_{\theta}} g\left(x^{2}+y^{2}\right) d x d y,
$$

where $a_{\theta}=(r \cos \theta, r \sin \theta)$, does not increase on $[0, \pi / 2]$.

Proof. The function $H(\theta)$ can be written as 


$$
\begin{gathered}
H(\theta)=\int_{(x-r \cos \theta, y-r \sin \theta) \in M} g\left(x^{2}+y^{2}\right) d x d y= \\
=\int_{(x, y) \in M} g\left(x^{2}+y^{2}+2 r(x \cos \theta+y \sin \theta)+r^{2}\right) d x d y .
\end{gathered}
$$

Differentiating this expression with respect to $\theta$ and denoting $x \cos \theta+$ $+y \sin \theta=s,-x \sin \theta+y \cos \theta=t$, we have

where

$$
H^{\prime}(\theta)=\int_{M_{\theta}} g^{\prime}\left(s^{2}+t^{2}+2 r s+r^{2}\right) t d s d t,
$$

$$
M_{\theta}=\left\{(s, t) \in R^{2}:(s \cos \theta-t \sin \theta, s \sin \theta+t \cos \theta) \in M\right\},
$$

the set being obtained by turning the set $M$ around its centre of symmetry in the negative direction by an angle $\theta$. Let us present the set $M_{\theta}$ as the union of two non-intersecting sets:

$$
M_{\theta}=\left\{M_{\theta} \cap M_{-\theta}\right\} \cup\left\{M_{\theta} \backslash\left(M_{\theta} \cap M_{-\theta}\right)\right\} .
$$

By Lemma $1^{*}$ the set $M_{\theta} \cap M_{-\theta}$ is symmetric relative to the axis $O S$, and hence,

$$
\int_{M_{\theta} \cap M_{-\theta}} g^{\prime}\left(s^{2}+t^{2}+2 r s+r^{2}\right) t d s d t=0 .
$$

The symmetry of $M_{\theta} \cap M_{-\theta}$ also implies that the set $M_{\theta} \backslash\left(M_{\theta} \cap M_{-\theta}\right)$ is symmetric relative to both axes $(O S)$ and $(O T)$ as a difference of symmetric sets. By Lemma 2 the points of the set $M_{\theta} \backslash\left(M_{\theta} \cap M_{-\theta}\right)$ have Cartesian coordinates with different signs, and hence,

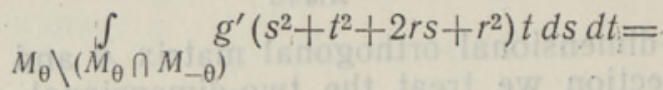

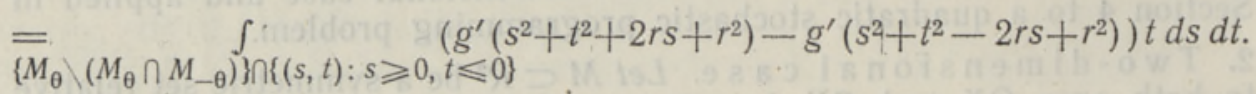

Since the function $g(\cdot)$ is convex and in the formula (3) $s \geqslant 0$, we have for $(s, t) \in M_{\theta} \backslash\left(M_{\theta} \bigcap M_{-\theta}\right)$ :

$$
g^{\prime}\left(s^{2}+t^{2}+2 r s+r^{2}\right) \geqslant g^{\prime}\left(s^{2}+t^{2}-2 r s+r^{2}\right) .
$$

Combining (2) and (3), we obtain by virtue of (4) that $H^{\prime}(\theta) \leqslant 0$. Q.E.D. Lemma 1 . Set $M_{\theta} \cap M_{-\theta}$ is symmetric relative to both axes, i.e. if $(s, t) \in M_{\theta} \cap M_{-\theta}$, then $(s,-t) \in M_{\theta} \cap M_{-\theta}$ and $(-s, t) \in M_{\theta} \cap M_{-\theta}$.

P r o of. Let $(s, t) \in M_{\theta} \cap M_{-\theta}$. Then by definition $(s \cos \theta-t \sin \theta$, $s \sin \theta+t \cos \theta) \in M$ and also $(s \cos \theta+t \sin \theta,-s \sin \theta+t \cos \theta) \in M$. By symmetry of the set $M$ relative to axis $O Y$ we get that $(s \cos \theta-t \sin \theta$, $-s \sin \theta-t \cos \theta) \in M$ and $(s \cos \theta+t \sin \theta, s \sin \theta-t \cos \theta) \in M$ meaning that $(s,-t) \in M_{\theta} \cap M_{-\theta}$, i. e. the set $M_{\theta} \cap M_{-\theta}$ is symmetric relative to the axis $O S$. Proof of the symmetry relative to the axis $O T$ is analogous.

Le $\mathrm{mm}$ a 2. The following equality holds:

$$
\left\{M_{\theta} \backslash\left(M_{\theta} \cap M_{-\theta}\right)=\left\{M_{\theta} \backslash\left(M_{\theta} \cap M_{-\theta}\right)\right\} \cap\left\{(s, t) \in R^{2}: \text { st }<0\right\} .\right.
$$

Proof. Equality (5) means that no point in the set $M_{\theta} \backslash\left(M_{\theta} \cap M_{-\theta}\right)$ with Cartesian coordinates has identical signs. From (1) we have

* The statement and proof of Lemmata 1 and 2 are presented at the end of the Section. 


$$
\begin{gathered}
M_{\theta}=\{(\varrho, \alpha): \varrho \leqslant \varphi(\alpha+\theta), \varrho \geqslant 0,0<\alpha<2 \pi\}, \\
M_{-\theta}=\{(\varrho, \alpha): \varrho \leqslant \varphi(\alpha-\theta), \varrho \geqslant 0,0<\alpha<2 \pi\},
\end{gathered}
$$

and hence,

$$
M_{\theta} \backslash\left(M_{\theta} \cap M_{-\theta}\right)=\{(\varrho, \alpha): \varphi(\alpha-\theta) \leqslant \varrho \leqslant \varphi(\alpha+\theta), \varrho \geqslant 0,0<\alpha<2 \pi\} .
$$

We shall show that for points with Cartesian coordinates with identical signs, i.e. in polar coordinates for $0<\alpha<\pi / 2$ and $\pi<\alpha<3 / 2 \pi$ the inequality

$$
\varphi(\alpha+\theta) \leqslant \varphi(\alpha-\theta)
$$

is valid and thus no such point belongs to $M_{\theta} \backslash\left(M_{\theta} \cap M_{-\theta}\right)$.

We shall prove (6) only for $0<\alpha<\pi / 2$, the rest of the cases being proved similarly. Note that $\varphi(\alpha+\theta)=\varphi(\alpha-\theta)$ for $\alpha=0$ and $\alpha=\pi / 2$. In addition, by virtue of strong prolation of $M$, the function

$$
\varphi(\alpha+\theta)\left\{\begin{array}{l}
\text { is non-increasing for } \alpha \in(0, \pi / 2-\theta), \\
\text { is non-decreasing for } \alpha \in(\pi / 2-\theta, \pi / 2),
\end{array}\right.
$$

and the function

$$
\varphi(\alpha-\theta)\left\{\begin{array}{l}
\text { is non-decreasing for } \alpha \in(0, \theta), \\
\text { is non-increasing for } \alpha \in(\theta, \pi / 2) .
\end{array}\right.
$$

This implies correctness of (6) for $\alpha \in(0, \theta) \cup(\pi / 2-\theta, \pi / 2)$. Now consider the interval $[\theta, \pi / 2-\theta]$. First we prove the inequality (6) for $\alpha \in[\theta, \min (2 \theta, \pi / 2-\theta)]$. Taking $\alpha^{*}=2 \theta-\alpha$, we get $2 \theta-\min (2 \theta, \pi / 2-$ $-\theta)<a^{*}<\theta$, but for such $\alpha^{*}$ the inequality (6) has already been proved. Hence $\varphi(\alpha-\theta)=\varphi\left(\alpha^{*}-\theta\right) \geqslant \varphi\left(\alpha^{*}+\theta\right) \geqslant \varphi(\alpha+\theta)$, because $\varphi(\alpha+\theta)$ is non-increasing for $\alpha \in(0, \pi / 2-\theta)$. Thus for $2 \theta>\pi / 2-\theta$. Lemma 2 is proved.

Consider the last case: $2 \theta<\pi / 2-\theta$. Here for $\alpha \in(0,2 \theta)$ holds $\varphi(\alpha-\theta) \geqslant \varphi(\alpha+\theta)$, while for $\alpha \in(2 \theta, \pi / 2-\theta)$ denoting $\alpha^{*}=\alpha-2 \theta$, we get

$$
\varphi(\alpha-\theta)=\varphi\left(\alpha^{*}+\theta\right) \geqslant \varphi(\alpha+\theta),
$$

since $\varphi(\alpha+\theta)$ is non-increasing in $(0, \pi / 2-\theta)$. This completes the proof of Lemma 2.

3. $n$-dimensional case. Here, we generalize the results of the previous section to the $n$-dimensional case. Define the strong prolation in $R^{n}$ by induction.

Definition 2. A set $M \subset R^{n} \quad(n>2)$ is called strongly prolate in $L$-direction if every $(n-1)$-dimensional section of the set $M$ parallel to the axis $L$, is strongly prolate in $L$-direction in $R^{n-1}$.

$\mathrm{Example.} \mathrm{The} \mathrm{ellipsoid} \mathrm{is} \mathrm{strongly} \mathrm{prolate} \mathrm{along} \mathrm{its} \mathrm{longest} \mathrm{axis.}$

Define

$$
R(a, A)=\int_{A M+a} g\left(x^{\mathrm{T}} x\right) d x,
$$

where $g(t), 0<t<\infty$, is a Lebesgue integrable function, matrix $A$ belongs to the set of $n \times n$ orthogonal matrices $Q^{n}$ and the vector $a$ belongs to the $n$-dimensional sphere $S^{n}(r)$ with the radius $r$.

Lemma 3. For every $b \in S^{n}(r), B \in Q^{n}$ and $r>0$ the following equalities hold 


$$
\max _{\substack{A \in Q^{n} \\ a \in S^{n}(r)}} R(a, A)=\max _{A \in Q^{n}} R(b, A)=\max _{a \in S^{n}(r)} R(a, B) .
$$

Proof. Prove the first equality. Let the first maximum in (9) be attained at $a_{0} \in S^{n}(r)$ and $A_{0} \in Q^{n}$. Then, using in (8) the change of variables $x=V y, V b=a_{0}, V \in Q^{n}$, we get

$$
\begin{gathered}
\max _{\substack{A \in Q^{n} \\
a \in S^{n}(r)}} R(a, A) \geqslant \max _{A \in Q^{n}} R(b, A)= \\
=\max _{A \in Q^{n}} \int_{V} \int_{y \in A M+b} g\left(y^{\mathrm{T}} y\right) d y \geqslant \int_{A_{0} M+a_{0}} g\left(y^{\mathrm{T}} y\right) d y=R\left(a_{0}, A_{0}\right),
\end{gathered}
$$

which implies the first equality in (9). The proof of the second one is analogous.

Theorem 2. Let $g(t)$ be a non-negative, convex and differentiable function on $(0, \infty)$ and let $M \subset R^{n}$ be strongly prolate in L-direction. Then for an arbitrary $r>0$ the following relation holds

$$
\max _{\substack{A \in Q^{n} \\ a \in S^{n}(r)}} R(a, A)=R\left(a_{0}, A_{0}\right),
$$

where the vector $a_{0}$ is parallel to some axis $L_{0}$, along which the set $A_{0} M$ is strongly prolate.

R e mark. By Lemma 3 the solution of the problem (10) is not unique. Proof. The proof is effected by induction, Theorem 1 being taken as a basis. Let Theorem 2 hold for $n=k-1, k \geqslant 3$. Prove the relation (10) for $n=k$. Choose matrix $A_{0}$ so that the set $A_{0} M$ would be strongly prolate in $O X$-direction. Let $a^{*}=\left(a_{1}^{*}, \ldots, a_{n}^{*}\right)$ be a solution of the problem (10). Then for $i \neq k$

$$
\begin{aligned}
& \max R\left(a, A_{0}\right)=\int d x_{i} \quad \int \quad g\left(x_{i}^{2}+\bar{x}_{i}^{\mathrm{T}} \bar{x}_{i}\right) d \bar{x}_{i}, \\
& \left.\left.a \in S^{n}(r) \quad\left(x_{t}-a_{i}\right)^{*}\right) \in P x_{t}^{\left(A_{0} M\right)} \quad \overline{(x}_{i}-\bar{a}_{i}\right) \in\left(A_{0} M\right) x_{t}
\end{aligned}
$$

where $\bar{x}_{i}=\left(x_{1}, x_{2}, \ldots, x_{i-1}, x_{i+1}, \ldots, x_{k}\right), \bar{a}_{i}^{*}=\left(a_{1}^{*}, a_{2}^{*}, \ldots, a_{i-1}^{*}, a_{i+1}^{*}, \ldots\right.$ $\left.\ldots, a_{k}^{*}\right), P_{X_{t}}\left(A_{0} M\right)$ is the projection of the set $A_{0} M$ to the $X_{i}$-axis and $\left(A_{0} M\right)_{x_{t}}$ is $(k-1)$-dimensional section of $A_{0} M$ with the plane $X_{i}=x_{i}$. By inductive assumption the integral

$$
\int_{\left(x_{t}-\bar{a}_{i}\right) \in\left(A_{0} M\right)_{x_{i}}} g\left(x_{i}^{2}+\bar{x}_{i}^{T} \bar{x}_{i}\right) d \bar{x}_{i}
$$

is maximized by the vector $b^{i}=\left(0, \ldots, 0, b_{i}, 0, \ldots, b_{k}\right)$ independently of the value of $x_{i}$ since strong prolation of the set $\left(A_{0} M\right)_{x_{t}}$ along the axis parallel to $X_{i}$-axis. Thus, the existence of such a coordinate $i \geqslant 1$, for which $\mathrm{a}_{i}^{*} \neq b_{i}$, contradicts $(11)$ and consequently $a_{i}^{*}=0, i=1,2, \ldots$ $\ldots, k-1, a_{k}^{*}=r$. Q.E.D.

4. Application to quadratic stochastic programming. Consider the following quadratic stochastic programming problem

$$
\max _{\substack{a \in A \\ D \in D_{\gamma}}} P\left[(X-a)^{\mathrm{T}} D(X-a) \leqslant 1\right],
$$


where $P$ is the symbol of probability, $A \subset R^{n}, D_{\gamma}$ is a class of positively defined $n \times n$-matrices such that the Lebesgue measure of the ellipsoid $\left\{X:(X-a)^{\mathrm{T}} D(X-a) \leqslant 1\right\}$ is bounded, i. e.

$$
\mu\left((X-a)^{\mathrm{T}} D(X-a) \leqslant 1\right) \leqslant \gamma,
$$

$X$ is a random vector with spherically symmetric distribution, i.e. the density function $p(\cdot)$ of $X$ exists and can be presented in the form

$$
p(x)=g\left(x^{\mathrm{T}} x\right),
$$

where $g(t)$ is convex and differentiable on $(0, \infty)$.

Denote by $0<\lambda_{1} \leqslant \lambda_{2} \leqslant \ldots \leqslant \lambda_{n}$ the eigen-values of the matrix $D$. Then by Theorem 2 for every $a \in A$

$$
\begin{gathered}
P\left[(X-a)^{\mathrm{T}} D(X-a) \leqslant 1\right]=\int_{\left(x-a^{*}\right)^{\mathrm{T}} C^{\mathrm{T}} \dot{D} C\left(x-a^{*}\right) \leqslant 1} g\left(x^{\mathrm{T}} x\right) d x \leqslant \\
\leqslant \int_{\lambda_{1}\left(x_{1}-\left\|a^{*}\right\|\right)^{2}+\sum_{i=2}^{n} \lambda_{t} x_{t}{ }^{2} \leqslant 1} g\left(x^{\mathrm{T}} x\right) d x,
\end{gathered}
$$

where $a^{*}=(\|a\|, 0, \ldots, 0)$ and $a^{*}=C a$ ( $C$ is an orthogonal matrix $)$. Correctness of the last inequality follows from the strong prolation of the ellipsoid $\left\{X:\left(X-a^{*}\right)^{\mathrm{T}} C^{\mathrm{T}} D C\left(X-a^{*}\right) \leqslant 1\right\}$ along the axis which is parallel to the eigen-vector of the matrix $C^{\mathrm{T}} D C$ corresponding to the eigen-value $\lambda_{1}$. From Lemma 1 in $\left[{ }^{4}\right]$ and conditions for $g(t)$ we get

$$
\int_{\lambda_{1}\left(x_{1}-\left\|a^{*}\right\|\right)^{2}+\sum_{i=2}^{n} \lambda_{t} x_{t}{ }^{2} \leqslant 1} g\left(x^{\mathrm{T}} x\right) d x \leqslant \int_{\lambda_{1}\left(x_{1}-r\right)^{2}+\sum_{i=2}^{n} \lambda_{t} x_{t}^{2} \leqslant 1} g\left(x^{\mathrm{T} x}\right) d x,
$$

where $r=\min _{a \in A}\|a\|$ and, consequently,

$$
P\left[(X-a)^{\mathrm{T}} D(X-a) \leqslant 1\right] \leqslant \max _{\lambda_{1} \leqslant \lambda_{2} \leqslant \cdots \leqslant \lambda_{n}} \int_{\lambda_{1}\left(x_{1}-r\right)^{2}+\sum_{i=2}^{n} \lambda_{t} x_{i}^{2} \leqslant 1} g\left(x^{\mathrm{T}} x\right) d x .
$$

Le $\mathrm{mm}$ a 4. For arbitrary $\lambda_{1}>0$ and $l>0$

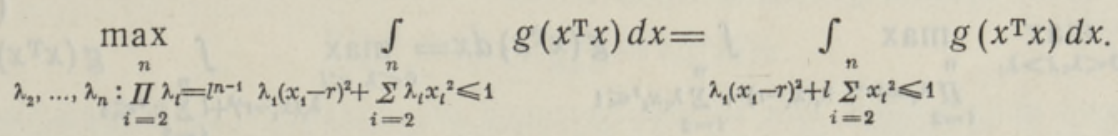

Proof. Consider two sets in $R^{n-1}$ :

$$
\begin{aligned}
Q_{\Lambda} & =\left\{y=\left(x_{2}, \ldots, x_{n}\right) \in R^{n-1}: y^{\mathrm{T}} \Lambda y \leqslant 1-\lambda_{1}\left(x_{1}-r\right)^{2}\right\}, \\
Q_{l} & =\left\{y=\left(x_{2}, \ldots, x_{n}\right) \in R^{n-1}: l y^{\mathrm{T}} y \leqslant 1-\lambda_{1}\left(x_{1}-r\right)^{2}\right\},
\end{aligned}
$$

where $\Lambda$ is a diagonal matrix with elements $\lambda_{2}, \ldots, \lambda_{n}$. Write the trivial expansion

$$
\begin{gathered}
\int_{n} g\left(x^{\mathrm{T}} x\right) d x= \\
=\int_{\lambda_{1}\left(x_{1}-r\right)^{2} \leqslant 1} d x_{1}\left(\int_{\lambda_{1}\left(x_{1}-r\right)^{2}+\sum_{i=2} \lambda_{l} x_{t}{ }^{2} \leqslant 1} g\left(x_{1}^{2}+y^{\mathrm{T}} y\right) d y+\int_{\left.Q_{\Lambda} \backslash{ }^{(Q)} \Lambda^{\prime} Q_{l}\right)} g\left(x_{1}^{2}+y^{\mathrm{T}} y\right) d y\right) .
\end{gathered}
$$


By the mean value theorem there exists a vector $y_{0} \in Q_{\Lambda} \backslash\left(Q_{\Lambda} \cap Q_{l}\right)$, so that $\int_{Q_{\Lambda} \backslash\left(Q_{\Lambda} \cap Q_{l}\right)} g\left(x_{1}^{2}+y^{\mathrm{T}} y\right) d y=g\left(x_{1}^{2}+y_{0}^{\mathrm{T}} y_{0}\right) \mu\left(Q_{\Lambda} \backslash\left(Q_{\Lambda} \cap Q_{l}\right)\right)$, and a vector $y_{1} \in Q_{l} \backslash\left(Q_{\Lambda} \cap Q_{l}\right)$, so that

$$
\int_{\left.Q_{l} \backslash Q_{\Lambda} \cap Q_{l}\right)} g\left(x_{1}^{2}+y^{\mathrm{T}} y\right) d y=g\left(x_{1}^{2}+y_{1}^{\mathrm{T}} y_{1}\right) \mu\left(Q_{l} \backslash\left(Q_{\Lambda} \cap Q_{l}\right)\right) .
$$

Note that the restriction $\prod_{i=2}^{n} \lambda_{i}=l^{n-1}$ means that for an arbitrary $\Lambda$ the Lebesgue measure $\mu\left(Q_{\Lambda}\right)$ of the ellipsoid $Q_{\Lambda}$ is constant, and, consequently,

$$
\mu\left(Q_{\Lambda} \backslash\left(Q_{\Lambda} \cap Q_{l}\right)\right)=\mu\left(Q_{l} \backslash\left(Q_{\Lambda} \cap Q_{l}\right)\right) .
$$

In addition, from the definition of the vectors $y_{0}, y_{1}$ and the sets $Q_{\Lambda}$, $Q_{l}$, we get

$$
y_{1}^{\mathrm{T}} y_{1} \leqslant l^{-1}\left(1-\lambda_{1}\left(x_{1}-r\right)^{2}\right)<y_{0}^{\mathrm{T}} y_{0} .
$$

Using (14)-(16), and non-decreasing property of the function $g(t)$, we obtain the inequality

$$
\int_{Q_{\Lambda} \backslash\left(Q_{\Lambda} \cap Q_{l}\right)} g\left(x_{1}^{2}+y^{\mathrm{T}} y\right) d y \leqslant \int_{Q_{l} \backslash\left(Q_{\Lambda^{\cap}} \cap Q_{l}\right)} g\left(x_{1}^{2}+y^{\mathrm{T}} y\right) d y,
$$

which with (14) proves Lemma 4.

From Lemma 4 we get

$$
\begin{aligned}
& \max _{0<\lambda_{1} \leqslant \ldots \leqslant \lambda_{n}} \int_{\substack{n \\
\lambda_{1}\left(x_{1}-r\right)^{2}+\sum_{i=2}^{n} \lambda_{2} x_{t}^{2} \leqslant 1}} g\left(x^{\mathrm{T} x} x\right) d x=
\end{aligned}
$$

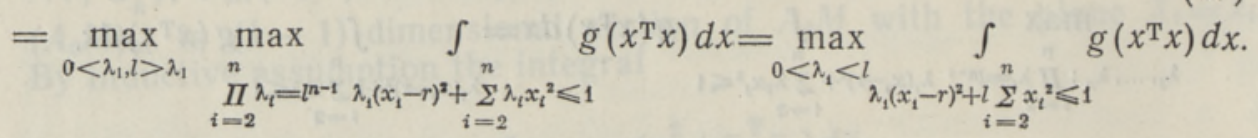

The restriction (13) is equivalent to the inequality

$$
\prod_{i=1}^{n} \lambda_{i} \geqslant \gamma^{-i} \Gamma^{n}(1 / 2) / \Gamma(n / 2+1)=q^{n-1},
$$

and, consequently, $\lambda_{1} l^{n-1} \geqslant q^{n-1}$. Denoting $\lambda_{1}=\lambda^{n-1}$ and using (17), we obtain the following form of the problem (12):

$$
\max _{\substack{0<\lambda<q^{1 / n} \\ \lambda^{n-1}\left(x_{1}-r\right)^{2}+q / \lambda}} \int_{\substack{\sum_{i=2} \\ x_{i}{ }^{2} \leqslant 1}} g\left(x^{\mathrm{T}} x\right) d x,
$$

i. e. the problem (12) is reduced to finding the maximum of a onedimensional function. 
REFERENCES

1. She rm a n, S., Ann. Math. Statistics, 26, № 4, 763-767 (1955).

2. Anderson, T. W., Proc. Amer. Math. Soc., 6, 170-176 (1955).

3. Mudholkar, G. S., Proc. Amer. Math. Soc., 17, 1327-1333 (1966).

4. О ль м а н В., Изв. АН ЭССР, Физ. Матем.. 23, № 2, 127-134 (1974).

Academy of Sciences of the Estonian SSR, Institute of Cybernetics

\title{
R. LEPP, V. OLMAN
}

\section{VÕRRATUS SFÄÄRILISELT SUMMEETRILISEST FUNKTSIOONIST SOLLTUVA INTEGRAALI KOHTA JA SELLE KASUTAMINE OPTIMEERIMISEKS}

On vaadeldud integraali väärtuse sōltuvust tugevalt väljavenitatud integreerimispiirkonna pööramisest oma tsentri ümber, kusjuures integreeritav funktsioon on sfääriliselt sümmeetriline. On näidatud, et integraali väärtus on maksimaalne, kui integreerimispiirkonna pikim telg ühtib piirkonna keskpunkti ja koordinaatide alguspunkti läbiva sirgega. Tulemust on kasutatud stohhastilise ruutplaneerimisülesande lahendi leidmisel.

\author{
Р. ЛЕПП, В. ОЛЬМАН
}

\section{НЕРАВЕНСТВО ДЛЯ ИНТЕГРАЛОВ ОТ СФЕРИЧЕСКИ СИММЕТРИЧНЫХ ФУНКЦИИ И ЕГО ПРИМЕНЕНИЕ В ОПТИМИЗАЦИИ}

Решается экстремальная задача, за критерий которой принимается значение интеграла от сферически симметричной функции по сильно вытянутому множеству с заданным центром, меняющееся при вращении множества вокруг центра. Доказано, что критерий достигает своего максимума, когда ось снльной вытянутости множества совпадает с прямой, проведенной из центра множества в начало координат. Полученный результат используется для нахождения решения задачи квадратичного стохастического программирования. 